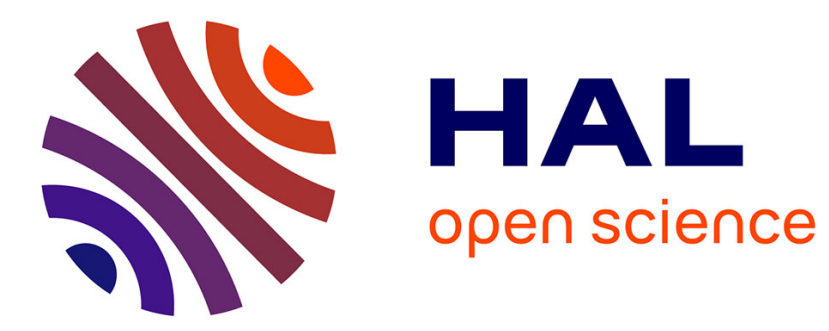

\title{
Unsupervised Stream-Weights Computation in Classification and Recognition Tasks
}

\author{
E. Sanchez-Soto, Alexandros Potamianos, Khalid Daoudi
}

\section{To cite this version:}

E. Sanchez-Soto, Alexandros Potamianos, Khalid Daoudi. Unsupervised Stream-Weights Computation in Classification and Recognition Tasks. IEEE Transactions on Audio, Speech and Language Processing, 2009, 17 (3), pp.436-445. inria-00438846

\section{HAL Id: inria-00438846 \\ https://hal.inria.fr/inria-00438846}

Submitted on 4 Dec 2009

HAL is a multi-disciplinary open access archive for the deposit and dissemination of scientific research documents, whether they are published or not. The documents may come from teaching and research institutions in France or abroad, or from public or private research centers.
L'archive ouverte pluridisciplinaire HAL, est destinée au dépôt et à la diffusion de documents scientifiques de niveau recherche, publiés ou non, émanant des établissements d'enseignement et de recherche français ou étrangers, des laboratoires publics ou privés. 


\title{
Unsupervised Stream-Weights Computation in Classification and Recognition Tasks
}

\author{
Eduardo Sánchez-Soto, Alexandros Potamianos, Member, IEEE, and Khalid Daoudi, Member, IEEE
}

\begin{abstract}
In this paper, we provide theoretical results on the problem of optimal stream weight selection for the two stream classification problem. It is shown that in the presence of estimation or modeling errors using stream weights can decrease the total classification error. Specifically, we show that stream weights should be selected to be proportional to the feature stream reliability and informativeness. Next, we turn our attention to the problem of unsupervised stream weights computation in real tasks. Based on the theoretical results we propose to use models and "anti-models" (class-specific background models) to estimate stream weights. A nonlinear function of the ratio of the inter- to intra-class distance is proposed for stream weight estimation. The resulting unsupervised stream weight estimation algorithm is evaluated on both artificial data and on the problem of audiovisual speech classification. Finally, the proposed algorithm is extended to the problem of audiovisual speech recognition. It is shown that the proposed algorithms achieve results comparable to the supervised minimumerror training approach for classification tasks under most testing conditions.
\end{abstract}

Index Terms-Decision fusion, multistream weights estimation, robust speech recognition.

\section{INTRODUCTION}

$\mathbf{T}$ HE problem of fusion or combination of various information sources is central to the machine learning community, especially, for signal processing and pattern recognition applications where a variety of features are available to the classifier. For example, for automatic speech recognition (ASR) and audiovisual speech recognition (AV-ASR) applications, the optimal combination of features extracted from the audio or visual data at different time scales is an open research problem. Features or information sources are often combined in a statistical pattern recognition framework using the notion of "feature streams." A fundamental assumption behind streams is that the information sources/features are independent of each other

Manuscript received January 28, 2008; revised November 28, 2008. Current version published February 11, 2009. The work of E. Sánchez-Soto was supported by the EU FP6-IST network of excellence "MUSCLE". The work of A. Potamianos and K. Daoudi was supported in part by the EU FP6-IST projects "HIWIRE" and "MUSCLE". The associate editor coordinating the review of this manuscript and approving it for publication was Prof. Mari Ostendorf.

E. Sánchez-Soto was with IRIT-CNRS, Toulouse 31062, France. He is now with France Telecom R\&D, 35512 Cesson Sévigné, France (e-mail: eduardo. sanchezsoto@orange-ftgroup.com; edusanso@gmail.com).

A. Potamianos is with the Department of Electronics and Computer Engineering, Technical University of Crete, Chania 73100, Greece (e-mail: potam@telecom.tuc.gr).

K. Daoudi is with IRIT-CNRS, Toulouse 31062, France (e-mail: daoudi@irit. fr).

Color versions of one or more of the figures in this paper are available online at http://ieeexplore.iee.org.

Digital Object Identifier 10.1109/TASL.2008.2011513 and thus the probability distribution functions (pdfs) of the two streams can be multiplied to obtain the joint observation pdf. Although this approach is theoretically sound because it minimizes the Bayes error, in the real world the independence assumption rarely holds. In addition, estimation and modeling errors further complicate the problem. It turns out that in the presence of estimation or modeling errors using the joint feature distribution in the Bayes classifier is suboptimal even for independent feature streams, i.e., although the Bayes error is minimized the total classification error (including errors due to poor estimation/modeling) might not be minimal. A practical solution to this problem is to use "stream weights" (exponents weighting the contribution of each stream pdf) in order to reduce the total classification error. Although these weights can be optimally computed in a supervised training setting using a minimum error criterion, the computation of the optimal weights in an unsupervised setting is still an open research problem.

The performance of speech recognition systems has improved significantly in the past decade. However, speech recognition in adverse or "mismatched" conditions is a hard problem, e.g., speech recognition in noise using acoustic models trained in "clean" conditions. As the signal-to-noise ratio (SNR) decreases, additional sources of information, e.g., visual information or noise-robust features, can be used to avoid performance loss. Such features are often combined with traditional Mel-frequency cepstrum coefficients (MFCCs) audio features using an information fusion method. Examples of information fusion methods that have been employed for speech processing applications can be found in the literature starting from early work on the combination of MFCCs with their first and second discrete time derivatives. Another example of a system that combines features with different reliabilities is the work on multiband ASR [1], where features extracted from certain frequency bands might be more (or less) affected by noise. In [2], features such as rate-of-speech (ROS) and fundamental frequency are used as auxiliary information for ASR. There is also much work in the area of audiovisual speech recognition where audio and visual features are fused; see for example [3]. Visual features have provided consistent ASR performance improvement especially in noisy or mismatched recording conditions.

The selected fusion strategy is characterized by the stage at which the information obtained from the different "modalities" is merged. The simplest approach is to fuse at the feature level. This technique, called early integration (EI), concatenates the features into a single feature vector before classification is performed [4], [5]. Feature selection or feature reduction algorithms, e.g., linear discriminant analysis (LDA), are often applied to reduce the dimension of and the dependencies within 
the feature vector for EI. Another approach is to perform integration at the decision level. In this approach, called late integration (LI), the classifier scores are combined assuming independence among the information sources. An important detail here is at which "level" the scores are combined, i.e., at the frame, word [6], [7] or utterance [5] level. A third approach, called middle integration (MI), allows the recognition system to define specific word or sub-word models and permits synchronous continuous speech recognition [8].

As discussed above, late and middle integration schemes may be suboptimal, both because the stream independence assumption rarely holds and because of the existence of estimation/modeling errors. In such cases, feature streams with higher reliability in the estimation process or informativeness (for the classification task) should be weighted more in the decision process in order to maximize performance. Therefore, a mechanism for weighting the stream contribution in the final decision is needed. Algorithms for computing exponential stream weights can be either supervised, i.e., assume that the speech transcription is known, or unsupervised. Unsupervised algorithms are especially relevant for mismatched training and testing conditions, or when the stream weights vary with time. In [9], the author proposes to use the static and dynamic features of the speech signal as two different streams, which are weighted based on a maximum-likelihood training algorithm under two different constraints. Given these constraints, the author reestimates the parameters by maximizing the partial Baum's auxiliary expression as a function of the weights. In [10]-[13], a generalized probabilistic descent (GPD) algorithm is used to estimate the stream weights using a minimum error classification criterion. Unsupervised algorithms often compute the stream weights based on reliability estimates of the environmental conditions, e.g., the SNR. In [14], the authors propose to weight dynamically the modalities as a function of the SNR and the phonetic content of the utterance. In [15], the authors present a decision fusion approach for AV-ASR, where the estimates of audio stream reliability and informativeness are based on the degree of voicing present in the utterance. In [7], it is assumed that the reliability and informativeness of each stream has a direct relation with the difference of the probability score among the first $N$ candidates produced by the recognizer. A similar approach is presented in [16]. In [17], the stream weights are estimated by minimizing the misclassification error on a held-out data set. Three stream confidence measures are investigated, namely the stream entropy, the $N$-best likelihood ratio average, and an $N$-best stream likelihood dispersion measure. Finally, in [18], stream weights are computed based on likelihood value normalization; weights are selected to maximize the difference between the $N$-best likelihood scores.

In this paper, we investigate the problem of unsupervised stream weight estimation with an application to audiovisual speech recognition. First, the stream weight estimation problem is posed as a probability of classification error minimization problem based on our prior work in [19]. These theoretical results are experimentally verified. Then, stream weights estimation algorithms based on the concept of "anti-models" are proposed extending the work in [20]. Extensive evaluation experiments are included that demonstrate the potential of these unsupervised stream weight estimation algorithms for classification and recognition problems. The contributions of this paper are 1) extensions to the theoretical framework of [19] and experimental verification of these results, and 2) theoretically motivated unsupervised stream weight estimation algorithms that extend the work of [20], along with detailed experimentation for the problem of AV-ASR.

The organization of this paper is as follows. In Section II, the theoretical underpinnings of the weighted multistream classification are presented. These results are experimentally evaluated in Section II-B. In Section III, an algorithm for estimating stream weights in an unsupervised manner is proposed and evaluated for the problem of audiovisual speech recognition. In Section IV, the proposed algorithm is extended to the problem of AV-ASR recognition and evaluated. The paper concludes with Section V.

\section{OPtimal StREAM-Weight COMPUTATION}

In [19], the two-class $w_{1}, w_{2}$, statistical classification problem with feature pdfs $p\left(x \mid w_{1}\right), p\left(x \mid w_{2}\right)$ and class priors $p\left(w_{1}\right), p\left(w_{2}\right)$ is presented. Based on the assumption that the estimation/modeling error for the feature pdfs is a random variable $z_{i}$, then the deviation of the decision boundary from the optimal Bayes boundary is also a random variable $z$ that is assumed zero-mean Gaussian with variance $\sigma^{2}$.

The classification decision is then a function of the random variable $z$ and the total classification error is computed as

$$
\begin{aligned}
P(\text { error })= & \int_{\mathbb{R}^{d}} \int_{f(x)}^{+\infty} \mathcal{N}\left(z ; 0, \sigma^{2}\right) d z p\left(x \mid w_{2}\right) p\left(w_{2}\right) d x \\
& +\int_{\mathbb{R}^{d}} \int_{-\infty}^{f(x)} \mathcal{N}\left(z ; 0, \sigma^{2}\right) d z p\left(x \mid w_{1}\right) p\left(w_{1}\right) d x
\end{aligned}
$$

where $f(x)=p\left(x \mid w_{2}\right) p\left(w_{2}\right)-p\left(x \mid w_{1}\right) p\left(w_{1}\right)$.

For multistream classification, we assume that the feature vector $x$ is broken up into two independent streams $x_{1}, x_{2}$ of dimension $d_{1}$ and $d_{2}$, respectively, and that the feature "probabilities" are given by

$$
p\left(x \mid w_{i}\right)=\prod_{j=1}^{2} p\left(x_{j} \mid w_{i}\right)^{s_{j}}
$$

where $s_{1}, s_{2}$ are the exponential stream weights, and $\sum_{j} s_{j}=$ 1 . Note that the total error (1) also holds for a two-stream classifier provided that $f(x)$ is substituted by

$$
f(x)=\prod_{j=1}^{2}\left[p\left(x_{j} \mid w_{2}\right) p\left(w_{2}\right)\right]^{s_{j}}-\prod_{j=1}^{2}\left[p\left(x_{j} \mid w_{1}\right) p\left(w_{1}\right)\right]^{s_{j}} .
$$

According to [19], since the total error functional in (1) cannot be minimized directly, an approximation is to compute weights that minimize the variance of the decision boundary deviation $\sigma^{2}$. By minimizing $\sigma^{2}$, the estimation/modeling error is minimized; however, there are no guarantees that the total 
error is also minimized. The deviation is shown to be a function of the stream weights as follows:

$$
\sigma^{2} \sim p\left(x_{1} \mid w_{1}\right)^{2 s_{1}} p\left(x_{2} \mid w_{1}\right)^{2 s_{2}}\left[s_{1}^{2} \sigma_{x_{1}}^{2}+s_{2}^{2} \sigma_{x_{2}}^{2}\right]
$$

where $\sigma_{x_{1}}^{2}, \sigma_{x_{2}}^{2}$ is the variance of the decision boundary deviation for each stream. The stream deviation variances can be expressed as a function of the estimation error variance as follows:

$$
\sigma_{x_{j}}^{2}=\sum_{i=1}^{2} \sigma_{i j}^{2}
$$

where $\sigma_{i j}^{2}$ is a variance of the estimation/modeling error for the posterior distribution of the $i$ th class and $j$ th stream. As noted in [19], from the equations above it follows that "stream weights may reduce estimation error only when either the pdf estimation errors of the single-stream (stand-alone) classifiers are different, i.e., one feature stream is more reliable than the rest, and/or the Bayes error of the single-stream classifiers are different, i.e., one stream contains more information pertinent to the classification problem than the rest".

It is also shown that if two streams have the same informativeness (equal Bayes classification errors), the stream weights are inversely proportional to the sum of the variances of the pdf estimation error for each of the classes of that given stream $\sigma_{x_{j}}^{2}$, i.e., proportional to a measure of the stream reliability as it is represented as follows:

$$
\frac{s_{1}}{s_{2}}=\frac{\sum_{i=1}^{2} \sigma_{i 2}^{2}}{\sum_{i=1}^{2} \sigma_{i 1}^{2}}=\frac{\sigma_{x_{2}}^{2}}{\sigma_{x_{1}}^{2}} .
$$

Similarly, if two streams are equally reliable (equal estimation error variances), the stream weights should be approximately inversely proportional to the classification error of the single stream classifiers, i.e., proportional to a measure of the stream informativeness. Specifically

$$
\frac{s_{1}}{s_{2}} \approx \frac{p\left(x_{2} \mid w_{1}\right)}{p\left(x_{1} \mid w_{1}\right)} \text { for } 0.67 \leq \frac{p\left(x_{1} \mid w_{1}\right)}{p\left(x_{2} \mid w_{1}\right)} \leq 1.5
$$

where $p\left(x_{1} \mid w_{1}\right)$ and $p\left(x_{2} \mid w_{1}\right)$ are the observation probabilities for the first and second streams close to the decision boundary.

Combining these two results, we conclude that: 1) stream weights should be proportional to the feature stream reliability and informativeness, 2) the inverse of the variance of the posterior probability estimate is a good measure of reliability, and 3 ) the inverse classification error is a good measure of informativeness.

\section{A. First-Order Correction}

The analysis of [19] is approximate in the sense that the selected weights do not minimize the total classification error but rather the decision boundary deviation variance $\sigma^{2}$. The use of nonequal stream weights however moves the decision boundary away from the Bayes decision boundary, i.e., the value that minimizes Bayes error. As a result, by selecting stream weights using the formulas proposed above, the estimation/modeling error will be minimized, but at the same the Bayes error will increase disproportionately. Thus, a first-order correction is needed so that the total error (sum of Bayes and estimation/modeling error) is minimized; this correction will bring the stream weights closer to the $s_{1}=s_{2}=0.5$ value.

The increase in Bayes error is approximately proportional to the change in the decision boundary, namely

$$
\begin{aligned}
& \left\{\prod_{j=1}^{2}\left[p\left(x_{j} \mid w_{2}\right) p\left(w_{2}\right)\right]^{s_{j}}-\prod_{j=1}^{2}\left[p\left(x_{j} \mid w_{1}\right) p\left(w_{1}\right)\right]^{s_{j}}\right\} \\
& -\left\{\prod_{j=1}^{2}\left[p\left(x_{j} \mid w_{2}\right) p\left(w_{2}\right)\right]^{0.5}-\prod_{j=1}^{2}\left[p\left(x_{j} \mid w_{1}\right) p\left(w_{1}\right)\right]^{0.5}\right\} .
\end{aligned}
$$

One could attempt the joint minimization of $\sigma^{2}$ and the quantity above, although the relative weighting of the two criteria is nontrivial to compute. Instead we observe that for Gaussian distributions the quantity above is approximately proportional to the deviation from equal weights, i.e., $s_{1}-0.5$ (or equivalently $0.5-s_{2}$ ), if we use the logarithmic discriminative function. Thus, for small deviations from equal weighting, a first-order correction factor could be applied that is a function of the deviation, as follows:

$$
\hat{s}_{j}=s_{j}-a\left(s_{j}-0.5\right)=(1-a) s_{j}+0.5 a
$$

where $s_{j}$ is the original stream weight estimate and $\hat{s}_{j}$ is the corrected one. The positive factor $a$ can be empirically estimated from experiments. Note, however, that for large deviations from equal weighting, higher order corrections might be needed.

\section{B. Numerical Simulations}

In this section, we experimentally verify the accuracy of the estimation process outlined above. For this purpose, we design a two-class two-stream classification experiment, where the features follow a Gaussian distribution. It is also assumed that there is a known estimation error ${ }^{1}$ for the mean or the variance of these Gaussian distributions. Since both the actual and estimated parameters of the distribution are known, the Bayes and total errors can be computed directly, either for equally weighted feature streams or, in general, for arbitrary stream weights $\left\{s_{1}, s_{2}\right\}$. Our goal is to compare the optimal stream weights, i.e., the stream weights that minimize the total classification error, with the stream weights computed using (6) and (7).

The two classes $w_{1}, w_{2}$ classification problem with two streams $x_{1}, x_{2}$ can be visualized in Fig. 1. For a given stream $x_{j}$, each class $w_{i}$ is modeled with a 1-D Gaussian distribution. The Bayes error in stream $x_{2}$ is shown as the shaded region (overlapping area under the two Gaussian distributions). The joint distributions, assuming independence between $x_{1}$ and $x_{2}$ and equal weights $s_{1}=s_{2}$, are also shown for classes $w_{1}$ and $w_{2}$.

For example, consider the two-stream two-class classification problem with the true distributions shown in Table I. The $a$ priori class probabilities are assumed equal. Note that the means and variances of the distributions have been selected so that the

\footnotetext{
${ }^{1}$ Alternatively, the estimation error could be negligible, and the difference in the actual and estimated parameters could be due to a mismatch between the training and testing conditions, i.e., a modeling error. In general, the errors will be due to both poor estimation and inaccurate modeling.
} 


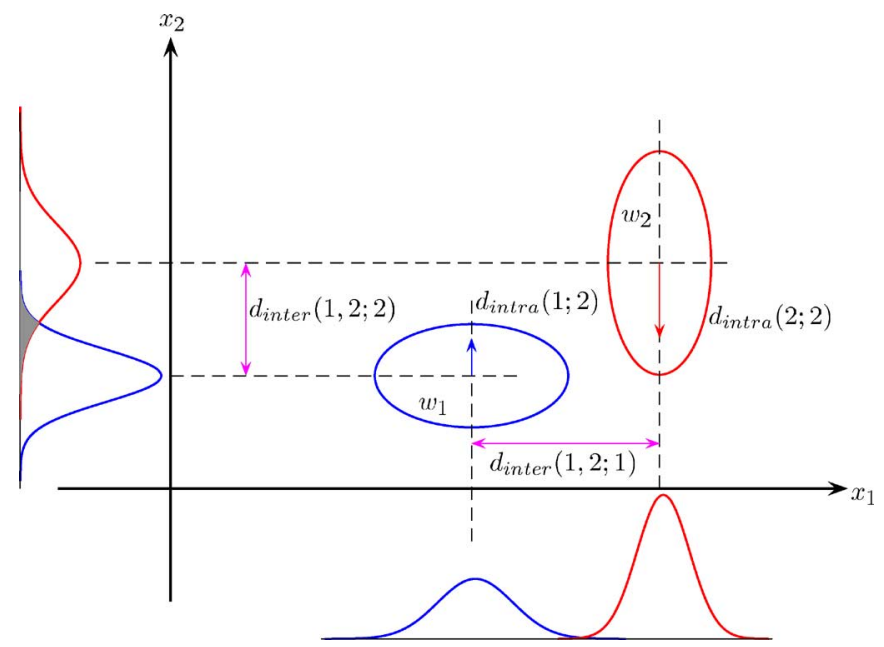

Fig. 1. Representation, in two dimensions, of the two classes $\left\{w_{1}, w_{2}\right\}$ classification problem. Each axis represents one stream $\left\{x_{1}, x_{2}\right\}$.

TABLE I

PARAMETERS FOR THE TWO-STREAM $\left\{x_{1}, x_{2}\right\}$ Two-Class $\left\{w_{1}, w_{2}\right\}$ GAUSSIAN DisTRIBUTIONS

\begin{tabular}{|c|c|c|c|c|c|}
\cline { 3 - 6 } \multicolumn{2}{c|}{} & \multicolumn{4}{c|}{ Class } \\
\cline { 3 - 6 } \multicolumn{2}{c|}{} & \multicolumn{2}{c|}{$w_{1}$} & \multicolumn{2}{c|}{$w_{2}$} \\
\cline { 2 - 6 } & $x_{1}$ & $\mu_{11}=4.0$ & $\sigma_{11}^{2}=2.0$ & $\mu_{21}=6.0$ & $\sigma_{21}^{2}=1.5$ \\
\cline { 2 - 6 } & $x_{2}$ & $\mu_{12}=1.5$ & $\sigma_{12}^{2}=1.5$ & $\mu_{22}=3.5$ & $\sigma_{22}^{2}=2.0$ \\
\hline
\end{tabular}

single stream Bayes error is equal for $x_{1}$ and $x_{2}$. This is equivalent to saying that $P_{1}$ (error) $=P_{2}$ (error), where the Bayes error for each stream is defined as

$$
P_{j}(\text { error })=\int_{\Omega_{1}} p\left(x_{j} \mid w_{2}\right) p\left(w_{2}\right) d x+\int_{\Omega_{2}} p\left(x_{j} \mid w_{1}\right) p\left(w_{1}\right) d x
$$

where $\Omega_{1}$ and $\Omega_{2}$ are the decision regions for $w_{1}$ and $w_{2}$, respectively.

Next, we demonstrate that in the presence of estimation (or modeling) errors, one can significantly reduce the total classification error by using stream weights. In the example shown here, it is assumed that errors exist only in the estimation of the means of the first stream $x_{1}$. The total error is computed using (10) and shown in Fig. 2 as a function of the stream weight $s_{1}$ $\left(s_{1}=0.5\right.$ corresponds to equal stream weights). Three total error curves are shown for estimation error 0,1 , and 3, respectively. As expected, equal weights achieve minimum error if there is no estimation error. However, in the presence of estimation errors, the (more) "corrupted" feature stream should be weighted less in the final decision in order to maximize performance. As shown, the higher the estimation error, the smaller the corresponding stream weight $s_{1}$ should be.

In the next experiment, we attempt to verify the results of (6). We assume that for each pdf $\mathcal{N}\left(\mu_{i j}, \sigma_{i j}^{2}\right)$ shown at Table I, the pdf estimation error is a random variable $z_{i j}$ that follows a Gaussian zero-mean distribution $\mathcal{N}\left(z_{i j} ; 0, \sigma_{z_{i j}}^{2}\right)$ according to the assumptions that lead to (6). The optimal weights are computed empirically by generating 100000 samples for each of the pdfs, computing the observation probability for each of the samples and adding random estimation errors to the computed probability that follow a zero-mean Gaussian distribution

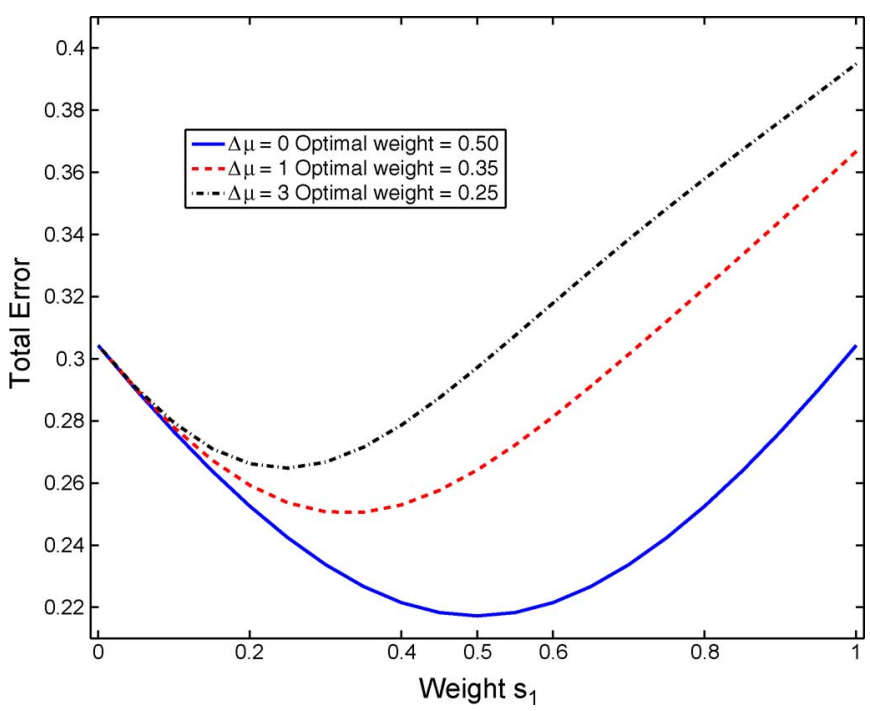

Fig. 2. Total error as a function of the "corrupted" stream weight $s_{1}$. Error curves are provided for absolute estimation errors 0,1 , and 3 .

TABLE II

OPTIMAL AND ESTIMATED WEIGHTS FOR $s_{1}$ AS A FUNCTION of (ACTUAL) PROBABILITY ERROR VARIANCE

\begin{tabular}{|c|c|c|c|c|c|c|}
\hline \multicolumn{4}{|c|}{ Probability Error Variance } & Optimal & Estimated & First Order \\
\hline$\sigma_{z_{11}}^{2}$ & $\sigma_{z 12}^{2}$ & $\sigma_{z 21}^{2}$ & $\sigma_{z 22}^{2}$ & Weight & Weight & Correction \\
\hline 0.01 & 0 & 0 & 0.01 & 0.50 & 0.50 & 0.50 \\
\hline 0.01 & 0 & 0 & 0.02 & 0.66 & 0.67 & 0.66 \\
\hline 0.01 & 0 & 0 & 0.03 & 0.73 & 0.75 & 0.73 \\
\hline 0.01 & 0 & 0 & 0.04 & 0.77 & 0.80 & 0.77 \\
\hline
\end{tabular}

with variance $\sigma_{z_{i j}}^{2}$. The optimal weights are then computed in order to maximize classification performance and compared with the estimated weights from (6). The results are shown at Table II for various probability error variances in the first and second streams. The last column of Table II shows the estimated weights following the application of the first-order correction shown in (9). The value of $a=0.085$ was chosen to minimize the mean square error between the estimated and optimal weights. Overall, there is good agreement between the optimal and estimated weights, especially after the first-order correction is applied.

In practice, however, estimation errors typically appear in the mean and, especially, the variances of the pdfs. Next, we experiment with various amounts of estimation errors in the variance of the Gaussian distributions of Table I. We use a stream weight estimate that is inversely proportional to the single stream classifier total error. Results are presented in Table III. In the first row of this table, the variances for all four pdfs are increased by the same amount $(+0.5)$, resulting in approximately the same pdf error variance and classification errors in both streams. Indeed for this case, both the optimal and estimated weights are equal to 0.5 . Then the variance $\sigma_{22}^{2}$ is increased further to create an imbalance in the estimation errors between the two streams. As expected, the weight for the less "corrupt" stream $s_{1}$ should then be higher to maximize performance. Note that there is good agreement between the optimal and estimated values for small estimation errors and stream weight ratios close to 1 . For large estimation errors, the approximation in (7) does not hold and 
TABLE III

OPTIMAL AND ESTIMATED WEIGHTS FOR $s_{1}$ AS A FUNCTION OF THE PDF VARIANCE ESTIMATION ERROR

\begin{tabular}{|c|c|c|c|c|c|}
\hline \multicolumn{4}{|c|}{ Variance Estimation Error } & \multirow{2}{*}{} \\
\cline { 1 - 3 }$\sigma_{11}^{2}$ & $\sigma_{12}^{2}$ & $\sigma_{21}^{2}$ & $\sigma_{22}^{2}$ & Optimal Weight & Estimated Weight \\
\hline+0.5 & +0.5 & +0.5 & +0.5 & 0.50 & 0.50 \\
\hline+0.5 & +0.5 & +0.5 & +1 & 0.53 & 0.53 \\
\hline+0.5 & +0.5 & +0.5 & +1.5 & 0.56 & 0.55 \\
\hline+0.5 & +0.5 & +0.5 & +2 & 0.60 & 0.58 \\
\hline+1 & +1 & +1 & +2 & 0.60 & 0.57 \\
\hline+1 & +1 & +1 & +3 & 0.70 & 0.62 \\
\hline
\end{tabular}
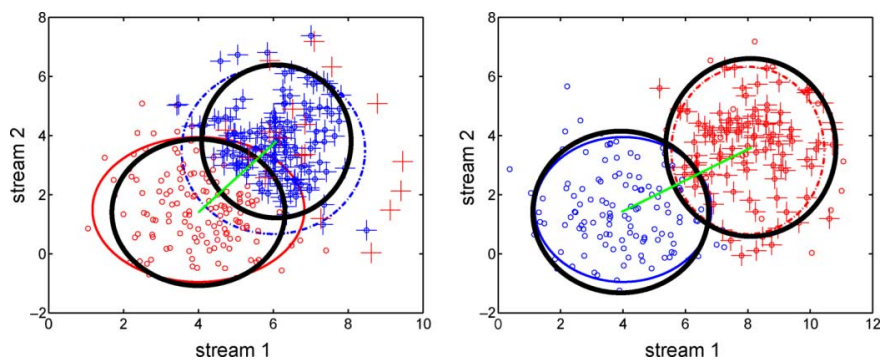

Fig. 3. Clustering and distance computation representation for the two class problem.

the difference between the actual and estimated weights is significant. In general, for cases where the informativeness and/or reliability of the two streams is very different further research is necessary to determine formulas for estimating the optimal stream weights.

\section{UNSUPERVISED STREAM WeIGHT ESTIMATION FOR CLASSIFICATION TASKS}

In real-world applications, we have no access to the true distribution but only to the estimated one. Hence, in this case the estimation/modeling error cannot be computed analytically. For example, in audiovisual speech recognition it is common that the recording conditions are both time-varying and different from the conditions under which the models were trained. In this case, the stream weights for the audio and video streams have to be adapted to their optimal values without knowledge of the transcription or "class labels." Our goal here is to devise a robust unsupervised estimation algorithm of the optimal stream weights using small amounts of unlabeled data and based on the theoretical results summarized in Section II. In this practical context, the theoretical results are not directly applicable because of two reasons: theoretical results are available only for the two-class classification problem, and for each observation $x$ the knowledge of class membership is required.

\section{A. Two-Class Problem}

It is well known that for the two-class classification problem, when $p\left(x \mid w_{i}\right)$ follow Gaussian distributions $\mathcal{N}\left(\mu_{i}, \sigma^{2}\right)$, the Bayes error is a function of $D=\left|\left(\mu_{1}-\mu_{2}\right)\right| / \sigma$. In general, the quantity $D$ can be estimated in an unsupervised way, by performing $k$-means classification and then using the inter- and intra-class distances to estimate the quantities in the nominator and denominator, respectively [20]. Indeed, the inter-class distance is the average distance between the means of each class and the intra-class distance an estimate of the average class variance.

To gain better insight into the use of the inter- and intra-class ratio, we display in Fig. 1 a two-stream two-class classification problem: axis $x_{1}$ and $x_{2}$ correspond to the features in the two streams; the (Gaussian) distributions for classes $w_{1}$ and $w_{2}$ are shown for each stream and jointly. The relationship between the Bayes error (shaded area) and the inter- and intra-class distances is approximately inversely and directly proportional, respectively.

Overall, the stream weights are computed using the interclass distance $d_{\text {inter }}(1,2 ; j)$ between classes 1 and 2 , normalized by the intra-class distance $d_{\text {intra }}(i ; j)$ for the class $i$ in each stream. Specifically

$$
\frac{s_{1}}{s_{2}}=c f\left(\frac{d_{\text {inter }}(1,2 ; j) /\left.\sum_{i} d_{\text {intra }}(i ; j)\right|_{j=1}}{d_{\text {inter }}(1,2 ; j) /\left.\sum_{i} d_{\text {intra }}(i ; j)\right|_{j=2}}\right)
$$

where $f($.$) is a nonlinear function that relates D$ with the Bayes error $(\operatorname{erf}()$ function) and $c$ is a constant accounting for the difference in estimation error in the two streams. ${ }^{2}$

1) Evaluation on Synthetic Data: In this experiment, we used the parameters shown in Table I for the 1-D Gaussian distributions of the two classes $\left\{w_{1}, w_{2}\right\}$. A number $N=250$ of samples was generated using those parameters and the total classification error was computed for different weights. The samples were used to estimate the distributions for the two classes by a clustering process. The $k$-means algorithm with $k=2$ was employed to cluster the samples. The estimated clusters were used to compute the distances as shown in (11). Results are shown in Fig. 3 for two examples. The solid (black) lines represent the estimated distributions, while the thin (blue and red) lines represent the real distributions. A solid (green) line connects the $k$-mean estimated centroids.

In the figure on the left, the optimal stream weight that minimized the total error was equal to 0.5 , while the estimated weight using the proposed approach was $s_{1}=0.47$ (using $c=1$ and $f()$ the identity function). In the figure on the right, one of the class means was moved from $\mu_{21}=6$ to $\mu_{21}=8$ to introduce additional modeling error. The optimal weight was in this case $s_{1}=0.6$ and the estimated one was 0.65 . Overall, for artificial data and for the two-class two-stream problem, the proposed approach gives satisfactory results.

\section{B. Multiclass Problem Using Anti-Models}

Another issue that has to be addressed is the generalization of the stream weight estimation process to multiple classes. Currently, theoretical results are available only for the two-class classification problem, while in general the multiclass classification problem is of interest.

To resolve the multiclass problem, we introduce the concept of anti-models. ${ }^{3}$ Specifically, during training and for each class, we separate the training data into two groups: one containing the training examples of the class of interest and the other containing the rest of the training examples.

\footnotetext{
${ }^{2}$ The quantity $D$ and the stream error are related through the $\operatorname{erf}()$ function only for the ideal case of Gaussian distributions. In general, this relationship might be nonlinear and can be approximated by a polynomial or sigmoid function estimated on held-out data (see also next section).
}

${ }^{3}$ Anti-digit models have been employed in utterance verification [21]. 
Models and "anti-models" are thus built from the two training sets; anti-models can be thought of as class-specific "background/garbage" models. By creating models and anti-models, the multiclass classification problem is reposed as multiple two-class classification problems.

\section{Application to Audiovisual Speech Classification}

To investigate the potential of our approach, a set of experiments using real data was performed. An audiovisual speech classification task was evaluated with the two feature streams containing audio and visual information, respectively.

For the purposes of this experiment the CUAVE audiovisual speech database was employed [22]. The subset of the CUAVE database used in these experiments consists of videos of 36 persons each uttering 50 connected digits utterances. The training set is made up of 30 speakers (1500 utterances) and the test set contains six speakers (300 utterances). The audio signal was corrupted by additive babble noise at various SNR levels; the video signal was clean in all experiments. The audio features used were the "standard" MFCC computed for frames with duration $20 \mathrm{~ms}$, extracted every $10 \mathrm{~ms}$. The acoustic vectors with dimension $d_{A}=39$, consist of 12-dimensional MFCCs, energy, and their first- and second-order derivatives. The visual features were extracted from the mouth region of each video frame by gray-scaling, down-sampling, and finally performing a 2-D discrete cosine transform (DCT). The first 13 most "energetic" DCT coefficients within the odd columns were kept [23], resulting in a video feature vector of dimension $d_{V}=39$ including the first- and second-order derivatives. Hidden Markov models (HMMs) were used for both acoustic and video model training. Context-independent whole-digit models with eight states per digit and a single Gaussian density distribution per state were used. Each one of the Gaussians is treated separately during the weight computation process. The HTK HMM toolkit was used for training each stream, audio and video, and also for testing (using HTK's built-in multistream capabilities).

An important part of the training process is the generation of "anti-models" [21]. The class and anti-class models are both built during the training phase using only "clean" data. The class model for each stream is created following the traditional training process. The anti-class models are trained using all the data that does not belong to the corresponding class. For example, the model for the digit one is created using all training data labeled as one, while the anti digit model one is trained using all the data not labeled as one. At the end of this process, 20 models are obtained for each stream, ten models for the digits (0-9) and ten anti-digits all with the same number of parameters.

During the test phase, these class and anti-class models are used to initialize the $k$-means classification algorithm. Specifically, the means of the Gaussian distribution in the class and anti-class model are used as the initial $k$-mean centroids. ${ }^{4}$ Given that $a$ priori it is not known to which class each utterance belongs, the features in each utterance are split into two classes

\footnotetext{
${ }^{4}$ It is important to remark that these anti-class models are only used to initialize the clustering process and that the models are trained using data recorded in "clean"conditions (different than the test conditions).
}

$(k=2)$ in ten different ways, one for each digit and antidigit model. ${ }^{5}$ The stream weights are estimated using (11). The inter- $d_{\text {inter }}$ and intra-class $d_{\text {intra }}$ distance is computed for each of the ten splits and the resulting inter- to intra-class ratio is averaged over the ten splits. Note that the stream weights are estimated for each utterance.

Specifically, the steps of the stream weight estimation algorithm are as follows. ${ }^{6}$ During training:

1) HMM models and anti-models are trained for each digit.

2) For each model and anti-model, the average MFCC vector is computed across all states (to be used for $k$-means initialization in testing).

During testing:

1) For each analysis frame of a test digit, the MFCC feature vectors are computed.

2) The $k$-means $(k=2)$ algorithm is run on the extracted MFCC feature vectors using ten different initializations, one for each model and anti-model pair.

3) The inter- and intra-class distances are computed for the two classes resulting from the $k$-means algorithm. The process is repeated for each of the ten different $k$-means initializations.

- The inter-class distance is computed as the Euclidean distance between the class centroids.

- The intra-class distance is computed as the average Euclidean pair-wise distance between all class members.

4) The ratio between the inter-class and sum of intra-class distances is computed for each stream and averaged over all ten $k$-means initializations.

5) The stream weights are computed using (11).

6) The computed weights are averaged over all digits in an utterance to come up with the per-utterance stream weight estimate.

In Fig. 4, the digit classification results are shown for various stream weight estimation algorithms. The thick solid curve (green) represents the results obtained searching by hand for the optimal values of the weights. The solid curve (black) uses equal weights in both streams (0.5). These two curves serve as reference and are used to evaluate our approach. The first (and crudest) stream weight estimate is shown with the dashed curve (red) and corresponds to (11) with $c=1$ and $f()$ being the identity function. To take into account the estimation error a constant $c$ can be estimated on held-out data and used to improve the results; this is represented with the dashed-dotted curve (blue). As seen in (11), the optimal weights are a nonlinear function $f()$ of the distances. The nonlinear transformation used here is $f(x)=x^{b}$, where $b$ is a parameter estimated on held-out data. For this set of experiments, $b=0.5$ and $f(x)=\sqrt{x}$. The dotted curve (magenta) shows the results obtained using this nonlinear transformation of the weights. This last curve provides a good match between the $D$ value and the Bayes error and results in performance comparable to the hand-picked optimal

\footnotetext{
${ }^{5}$ Note that phone and (especially) word-models consist of multiple states with different acoustic characteristics. If each state is considered a separate class, one could end-up building HMM state and anti-state models. Although such an approach has its merits, in this work, we treat all states of an HMM model as a single class and build word and anti-word models. The approach is simpler and the (state-level) segmentation problem is avoided.

${ }^{6}$ For simplicity, the processing steps for the audio stream are outlined next. Note that the same steps have to be followed also for the visual stream.
} 


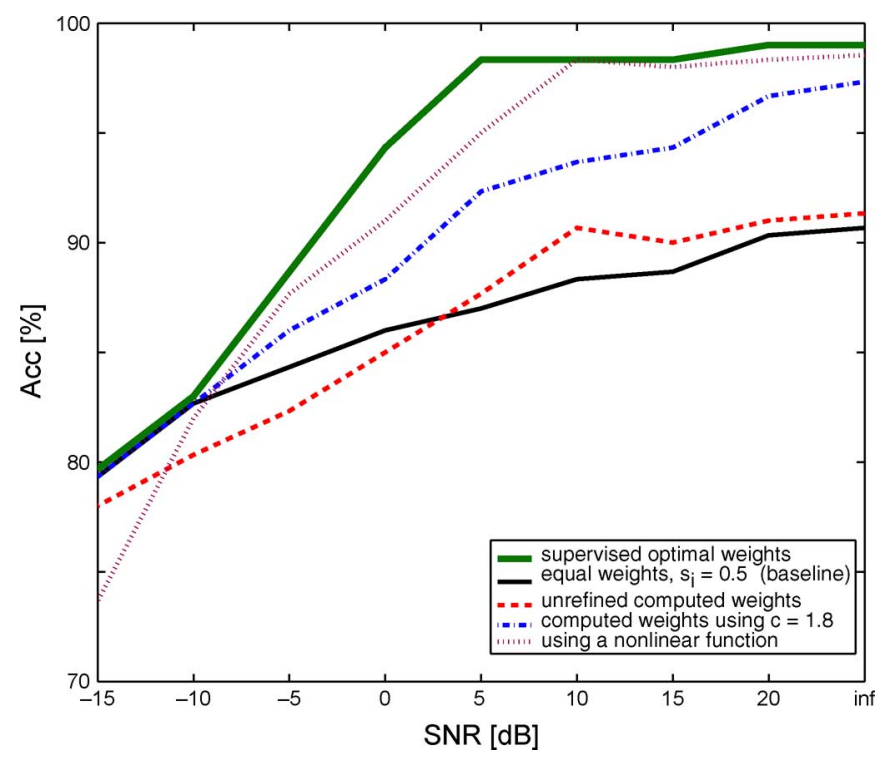

Fig. 4. Digit accuracy (optimal supervised, baseline, and obtained with the proposed method) as a function of SNR for the audiovisual digit classification task.

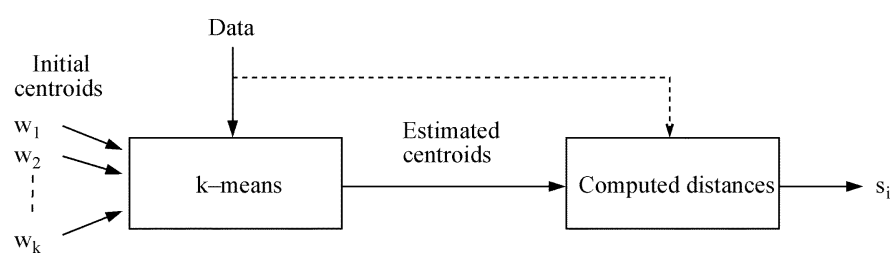

Fig. 5. Stream weight estimation process.

stream weight values, with the exception of the $-15-\mathrm{dB}$ SNR data point.

\section{UNSUPERVISED STREAM WEIGHT COMPUTATION FOR RECOGNITION TASKS}

In this section, we investigate the problem of unsupervised stream weight estimation for audiovisual speech recognition. Although recognition is in principle a more difficult problem than classification, our extension makes it actually easier to implement stream weight computation and without the need of anti-models. $^{7}$

We do so based on the observation that larger separation between class distributions in a given stream implies better discriminative power. Concretely, the inter-class distance is computed among all the classes by summing up all pair-wise interclass distances; the total inter-class distance measures the average separation between classes. ${ }^{8}$ The intra-class distances are computed as before to obtain an estimate of the class variance. The inter- and intra-class distances are combined to yield an estimate of the misclassification error for each stream.

\footnotetext{
${ }^{7}$ In order to use anti-models previously, we assumed that the digit segmentation boundaries are known. One could compute approximately the digit segmentation boundaries in a first recognition pass and employ the algorithm proposed in Section III also for recognition problems. Such a two-pass approach is beyond the scope of this paper; herein, we investigate unsupervised stream weight estimation for single pass recognition.

${ }^{8}$ Note that, as before, each HMM model is considered a single class (alternatively each HMM state can be considered a separate class).
}

As delineated in the previous section the initial centroids are obtained directly from the (HMM) models learned in training. This time the $k$-means algorithm is performed over all the classes only one time ( $k$ this time is the number of HMM models). The total inter-class distance $d_{\text {inter }}(j)$ is computed by summing the inter-class distance over all the possible combinations of two classes, as follows:

$$
d_{\text {inter }}(j)=\sum_{i=1}^{k} \sum_{l=i+1}^{k} d_{\text {inter }}(i, l ; j)
$$

where $d_{\text {inter }}(i, l ; j)$ is the inter-class distance between class $i$ and $l$ of stream $j$, and $k$ is the total number of classes.

Finally, the stream weights are computed using the total interclass distance $d_{\text {inter }}(j)$ normalized by the sum of the intra-class distances $d_{\text {intra }}(i ; j)$ in the corresponding stream $j$, as follows:

$$
\frac{s_{1}}{s_{2}}=c f\left(\frac{d_{\text {inter }}(j) /\left.\sum_{i=1}^{k} d_{\text {intra }}(i ; j)\right|_{j=1}}{d_{\text {inter }}(j) /\left.\sum_{i=1}^{k} d_{\text {intra }}(i ; j)\right|_{j=2}}\right)
$$

where $c$ is a constant and $f()$ is a nonlinear function.

The stream weight estimation algorithm, also shown in Fig. 5, is summarized next. During training:

1) For each HMM model, the average MFCC vector is computed across all model states (to be used for $k$-means initialization in testing). The process is repeated also for the visual stream.

During testing:

1) For each utterance, the MFCC feature vectors are computed.

2) The $k$-means algorithm is run on the extracted MFCC feature vectors ( $k$ equals number of HMM models, i.e., $k=$ 10 for digit recognition).

3) The inter- and intra-class distances are computed for the $k$ classes resulting from the $k$-means algorithm (see (12)).

4) Steps 1-3 are repeated for the visual stream and then the stream weights are computed using (13).

\section{A. Application to Audiovisual Speech Recognition}

A set of experiments using real data was performed to evaluate the proposed stream weight estimation algorithm. As before, a two stream audiovisual recognition task was investigated using the CUAVE audiovisual speech database [22]. The audio signal was corrupted by additive babble noise at various SNR levels; the video signal was clean in all the experiments. The results are given as a function of the audio SNR (\{infinity $20151050-5-10-15\}$ all given in $\mathrm{dB}$ ). The front-end, HMM topology and train/test data split were identical to those used in the classification experiment. It is important to remark that clean data were used in this experiment for both HMM training and $k$-mean centroid initialization. Only two iterations of the $k$-means algorithm were performed to compute the new centroids.

The digit accuracy as a function of audio stream SNR for various stream weight estimation methods is presented in Fig. 6. The thick solid curve (green) represents the results obtained searching (by hand) in a supervised manner for the optimal weight values. The solid curve (black) uses equal weights in both streams $\left(s_{1}=s_{2}=0.5\right)$. These two curves 


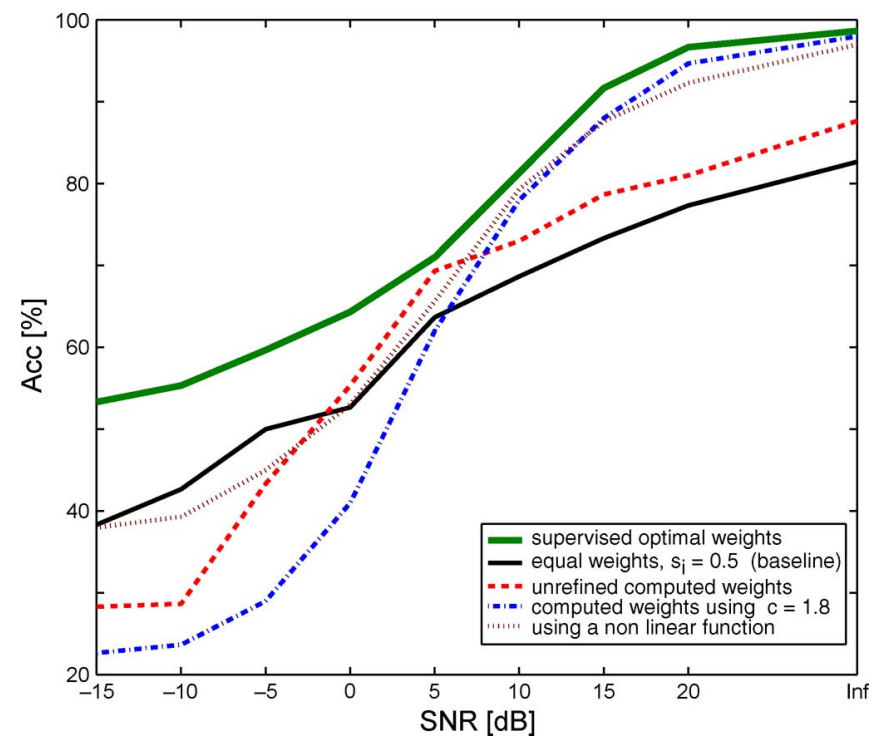

Fig. 6. Digit accuracy (optimal supervised, baseline and obtained with the proposed method) as a function of SNR for the audiovisual digit recognition task.

serve as reference and are used to evaluate our approach. ${ }^{9}$ The first (and crudest) stream weight estimate is shown with the dashed curve (red) and corresponds to (13) with $c=1$ and $f()$ being the identity function. Even this crude estimate improves the equal weighting scheme over most SNR values. To take into account the estimation error, a constant $c=1.8$ was estimated on held-out data. The corresponding word accuracy for $c=1.8$ and $f()$ being the identity function is shown with the dashed-dotted curve (blue). Good performance is achieved over $10-\mathrm{dB}$ SNR. However, below $10 \mathrm{~dB}$, the performance is poor, which shows that (as suggested by the theory and observed in classification) a nonlinear mapping function $f()$ is needed for low SNRs. This is confirmed by the word accuracy results shown with a dotted (magenta) curve, where the non linear function $f(x)=\sqrt{x}$ is used. One can see that performance improves further, especially for low SNRs. Finally, we tested the first-order weight correction shown in (9) with $a=0.085$ (not shown in the plot). A small improvement in performance was obtained for low SNR values, but the improvement was not significant. Overall, the results are satisfactory but not as good as the ones obtained for classification tasks.

One probable explanation for the worse performance of the stream weight estimation algorithm for recognition is the increased number of classes used by the $k$-means algorithm, i.e., by using models and anti-models $k=2$ for classification tasks, while for digit recognition $k=10$ (or in general $k$ is equal to the number of HMM models). Next, we show that indeed the stream weight estimation algorithm is sensitive to the initial choice of $k$-means centroids. The initial centroids are obtained directly from the mean values of the pdfs learned during training. These pdfs are estimated on clean data; thus, for low SNR values there is significant mismatch between the computed centroids and the actual data used by the $k$-mean algorithm. To

\footnotetext{
${ }^{9}$ When making comparisons between Figs. 4 and 6, bear in mind that results shown are from classification and recognition experiments, respectively. The significantly lower performance for mid and low SNRs in Fig. 6 is due to the many insertions that occur in the visual stream recognizer.
}

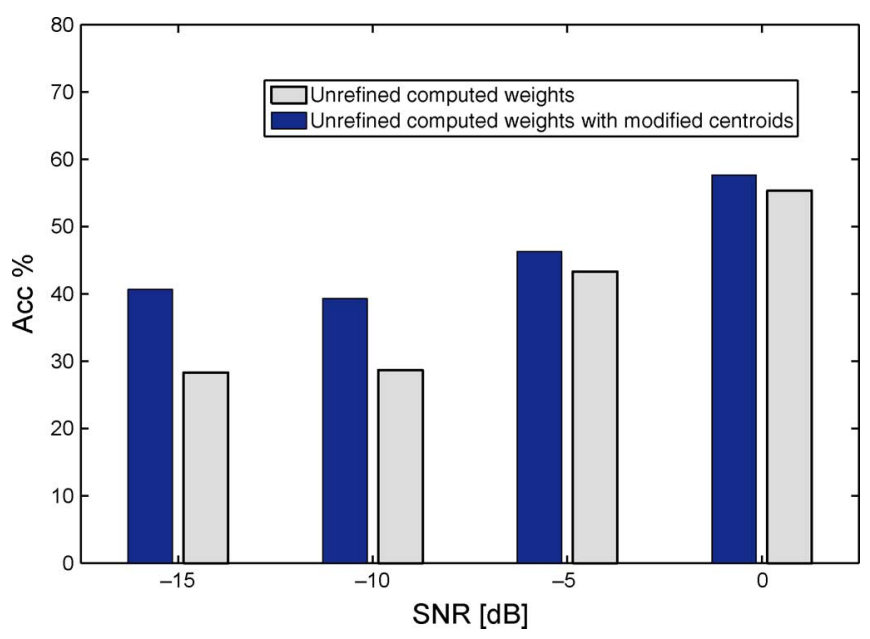

Fig. 7. Speech recognition accuracy as a function of SNR using $k$-means initialization from clean data training (unrefined weights) and "correct" $k$-mean centroids (unrefined weights with modified centroids).

investigate the importance of the $k$-means initialization, we perform an experiment where the "correct" centroids are used to compute the inter- and intra-distances (and the stream weights). Specifically, we assume that the boundaries of each digit and the digit transcription are known for the test data; the centroid for each class is then computed using the average MFCC vector of each digit occurrence. The results are shown in Fig. 7. The digit accuracy obtained using the "correct" centroids for stream weight estimation (labeled "modified centroids") is compared with the performance of the proposed algorithm. Note that for both cases the centroids are computed on the same test data, the only difference is the initialization of the $k$-means algorithm.

For all SNR values, the weights estimated using the "correct" centroids outperform, in terms of digit accuracy, the weights estimated using the proposed unsupervised stream weight estimation algorithm. We observe that the difference between the two methods is small for high SNR values, while the difference is significant for low SNRs. Based on these results, it is clear that the initialization of the $k$-means algorithm plays an important role, i.e., poor initialization results in worse performance. In addition, the performance gap increases for low SNR where the mismatch between the "correct" and initialized (from clean data) centroids is higher. It is important to remark that the centroids are just used to compute the stream weights and that the model pdfs are not modified in this experiment.

\section{CONCLUSION}

In this paper, we have presented theoretical and experimental results for the problem of optimal stream weight computation for multistream classification and recognition. It was shown that stream weights should be proportional to the feature stream reliability and informativeness to optimize classification performance. Metrics of reliability and informativeness were derived theoretically and tested experimentally. Then, a fully unsupervised method for computing stream weights was proposed making use of an "anti-model" technique. The proposed method employs only the information contained in the trained models and requires a single utterance to compute the stream weights. The proposed method achieved comparable performance with supervised minimum error estimation of the weights. Finally, 
the problem of stream weight estimation for recognition was addressed with good stream weight estimation results; although, the performance is worse than for the classification problem where anti-models were employed.

The results are encouraging but more research work is needed on both the theoretical and algorithmic front. Ongoing work includes the extension of the theoretical results to multi-class classification and recognition problem, improved criteria for performing clustering, the use of acoustic model adaptation to improve $k$-mean centroids initialization, as well as, the extension of the algorithm for the computation of time-varying stream weights.

\section{ACKNOWLEDGMENT}

The authors would like to thank I. Rodomagoulakis for independently reproducing the results in this paper using different visual front-end and recognizer setups.

\section{REFERENCES}

[1] H. Bourlard and S. Dupont, "A new ASR approach based on independent processing and recombination of partial frequency bands," in Proc. ICSLP, Philadelphia, PA, Oct. 1996.

[2] T. Stephenson, M. Mathew, and H. Bourlard, "Modeling auxiliary information in bayesian network based ASR," in Proc. Eurospeech, Aalborg, Denmark, Sep. 2001.

[3] G. Potamianos, C. Neti, G. Gravier, A. Garg, and A. Senior, "Recent advances in the automatic recognition of audiovisual speech," Proc. IEEE, vol. 91, no. 9, pp. 1306-1326, Sep. 2003.

[4] C. Chibelushi, J. Mason, and F. Deravi, "Integration of acoustic and visual speech for speaker recognition," in Proc. EUROSPEECH, Berlin, Germany, Sep. 1993.

[5] S. Dupont and J. Luettin, "Audio-visual speech modeling for continuous speech recognition," IEEE Trans. Multimedia, vol. 2, no. 3, pp. 141-151, Sep. 2000.

[6] M. Heckmann, F. Berthommier, and K. Kroschel, "Noise adaptive stream weighting in audio-visual speech recognition," EURASIP $J$. Appl. Signal Process., vol. 1, no. 11, pp. 1260-1273, Nov. 2002.

[7] A. Adjoudani and C. Benoit, "On the integration of auditory and visual parameters in an HMM-based ASR," Springer Verlag, Series F: Comput. Syst. Sci., vol. 150, pp. 465-472, 1996.

[8] J. Luettin, G. Potamianos, and C. Neti, "Asynchronous stream modeling for large vocabulary audio-visual speech recognition," in Proc. ICASSP, Salt Lake City, UT, May 2001, pp. 169-172.

[9] J. Hernando, "Maximum likelihood weighting of dynamic speech features for CDHMM speech recognition," in Proc. ICASSP, Munich, Germany, Apr. 1997, pp. 1267-1270.

[10] G. Potamianos and H. P. Graf, "Discrimative training of HMM stream exponents for audio-visual speech recognition," in Proc. ICASSP, Seattle, WA, May 1998, pp. 3733-3736.

[11] C. Miyajima, K. Tokuda, and T. Kitamura, "Audio visual speech recognition using MCE-based HMMs and model dependent stream weights," in Proc. ICSLP, Beijing, China, Oct. 2000.

[12] G. Potamianos, J. Luettin, and C. Neti, "Hierarchical discriminant features for audio-visual LVCSR," in Proc. ICASSP, Salt Lake City, UT, May 2001, pp. 165-168.

[13] S. Nakamura, K. Kumatani, and S. Tamura, "Robust bi-modal speech recognition based on state synchronous modeling stream weight optimization," in Proc. ICASSP, Orlando, FL, May 2002, pp. 309-312.

[14] A. Rogozan, P. Deléglise, and M. Alissali, "Adaptive determination of audio and visual weights for automatic speech recognition," in Proc. Workshop Audio-Visual Speech Process., Rhodes, Greece, Sep. 1997.

[15] H. Glotin, D. Vergyri, C. Neti, G. Potamianos, and J. Luettin, "Weighting schemes for audio-visual fusion in speech recognition," in Proc. ICASSP, Salt Lake City, UT, May 2001.

[16] S. Nakamura, H. Ito, and K. Shikano, "Stream weight optimization of speech and lip image sequence for audio-visul speech recognition," in Proc. ICSLP, Beijing, China, Oct. 2000G. Potamianos and C. Neti, "Stream confidence estimation for audio-visual speech recognition," in Proc. ICSLP, Beijing, China, Oct. 2000.
[17] G. Potamianos and C. Neti, "Stream confidence estimation for audiovisual speech recognition," in Proc. ICSLP, Beijing, China, Oct. 2000.

[18] S. Tamura, K. Iwano, and S. Furui, "A stream-weight optimization method for multi-stream HMMs based on likelihood value normalization," in Proc. ICASSP, Philadelphia, PA, Mar. 2005, pp. 469-472.

[19] A. Potamianos, E. Sánchez-Soto, and K. Daoudi, "Stream weight computation for multi-stream classifiers," in Proc. ICASSP, Toulouse, France, May 2006, pp. 353-356.

[20] E. Sánchez-Soto, A. Potamianos, and K. Daoudi, "Unsupervised stream weight computation using anti-models," in Proc. ICASSP, Honolulu, HI, Apr. 2007, pp. 365-368.

[21] M. Rahim, C. Lee, and B. Juang, "Discriminative utterance verification for connected digits recognition," IEEE Trans. Speech Audio Process., vol. 5, no. 3, pp. 266-277, May 1997.

[22] E. Patterson, S. Gurbuz, Z. Tufekci, and J. N. Gowdy, "CUAVE: A new audio-visual database for multimodal human-computer interface research," in Proc. ICASSP, Orlando, FL, 2002, pp. 2017-2020.

[23] G. Potamianos and P. Scanlon, "Exploiting low face symmetry in appearance-based automatic speechreading," in Proc. Workshop AudioVisual Speech Process., British Columbia, Canada, Jul. 2005.

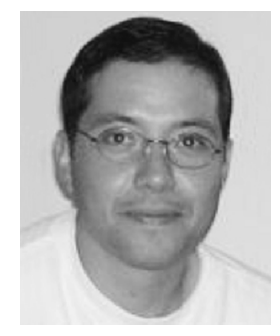

Eduardo Sánchez-Soto received the Engineering degree in telecommunications from the National $\mathrm{Au}-$ tonomous University of Mexico (UNAM), Mexico City, in 1999, the M.S. (DEA) degree in signal processing from the University of Rennes I, Rennes, France, in 2001, and the Ph.D. degree in images and signal processing from the National Superior School of Telecommunications (ENST), Paris, France, in 2005.

From 2005 to 2007, he was a Postdoctoral Fellow in the Department of Electronics and Computer Engineering, Technical University of Crete, Greece, and then at the Research Institute in Informatics of Toulouse (IRIT), Toulouse, France, where he worked on multimodal data fusion and language recognition. Currently, he is with the Research and Development Department, France Telecom, Cesson, Sévigné, France, working in audio quality measurement. His research interests lie mainly in speech processing and statistical modeling.

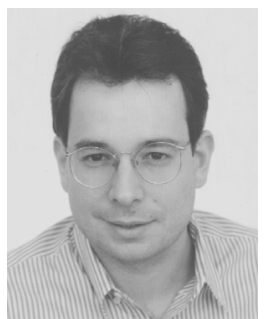

Alexandros Potamianos (M'92) received the Diploma in electrical and computer engineering from the National Technical University of Athens, Athens, Greece, in 1990 and the M.S. and Ph.D. degrees in engineering sciences from Harvard University, Cambridge, MA, in 1991 and 1995, respectively.

From 1991 to June 1993, he was a Research Assistant at the Harvard Robotics Lab, Harvard University. From 1993 to 1995 , he was a Research Assistant at the Digital Signal Processing Lab, Georgia Institute Technology, Atlanta. From 1995 to 1999, he was a Senior Technical Staff Member at the Speech and Image Processing Lab, AT\&T Shannon Labs, Florham Park, NJ. From 1999 to 2002, he was a Technical Staff Member and Technical Supervisor at the Multimedia Communications Lab at Bell Labs, Lucent Technologies, Murray Hill, NJ. From 1999 to 2001, he was an adjunct Assistant Professor at the Department of Electrical Engineering, Columbia University, New York. In the spring of 2003, he joined the Department of Electronics and Computer Engineering, Technical University of Crete, Chania, Greece, as an Associate Professor. His current research interests include speech processing, analysis, synthesis and recognition, dialog and multimodal systems, nonlinear signal processing, natural language understanding, artificial intelligence, and multimodal child-computer interaction. He has authored or coauthored over 80 papers in professional journals and conferences. He is the coeditor of the book Multimodal Processing and Interaction: Audio, Video, and Text (Springer, 2008). He holds four patents.

Prof. Potamianos received the 2005 IEEE Signal Processing Society Best Paper Award as coauthor of the paper "Creating conversational interfaces for children." He has been a member of the IEEE Signal Processing Society since 1992, and he is currently serving his second term at the IEEE Speech Technical Committee. 


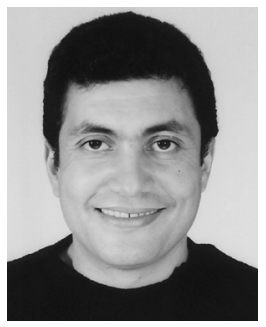

Khalid Daoudi (M'07) received the D.E.A. and $\mathrm{Ph} . \mathrm{D}$. degrees in applied mathematics from University Paris 9 Dauphine in 1993 and 1996, respectively. His Ph.D. dissertation was prepared at the Fractals Group of INRIA Rocquencourt, France.

During 1997, he held a postdoctoral position at the Department of Mathematics, Ecole Polytechnique de Montréal, Montreal, QC, Canada. From December 1997 to July 1999 , he held a postdoctoral position at the Stochastic Systems Group (SSG) of the Laboratory for Information and Decision Systems (LIDS), Massachusetts Institute of Technology (MIT), Cambridge. Since October 1999, he has had a permanent position at INRIA Lorraine within the Speech Group. Since October 2003, he is on leave at CNRS with the SAMOVA team of IRIT. His research interests include statistical modeling and estimation, machine learning, Bayesian networks, and speech and speaker recognition. 